\title{
CIRCUMNUCLEAR EMISSION IN NEARBY, NON-INTERACTING SEYFERT GALAXIES
}

\author{
Richard W. Pogge \\ Lick Observatory \\ University of California \\ Santa Cruz, CA 95064 \\ USA
}

\begin{abstract}
An investigation of the physical conditions present in the circumnuclear regions of 20 nearby, non-interacting Seyfert galaxies is reported. Significant differences are observed between the circumnuclear properties of Seyfert $1 \mathrm{~s}$ and Seyfert $2 \mathrm{~s}$ with regards to extended ionized gas. The morphology and ionization of the circumnuclear gas suggests collimation of the nuclear ionizing radiation field; in 4 cases taking the form of distinct conical distributions of ionized gas emanating from the nucleus. Some of these properties are consistent with prevailing "unified" models for AGN. However, it is difficult to reconcile all of the observed differences between Seyfert $1 \mathrm{~s}$ and $2 \mathrm{~s}$ with this picture.
\end{abstract}

\section{Observations}

CCD interference-band images isolating the bright emission lines of $\mathrm{H} \alpha+[\mathrm{N}$ II $]$ and [O III] $\lambda 5007$ were obtained to search for spatially extended circumnuclear emission regions in the 20 program galaxies. The starlight contribution was subtracted using continuumband images of adjacent spectral regions to produce essentially pure emission-line images. Long-slit, low-resolution spectrophotometry of interesting cases was used to probe the ionization state of the extended emission regions. All of the observations were carried out on the 1-m Nickel and 3-m Shane telescopes at Mt. Hamilton, California (see Pogge 1988 for details).

\section{Results}

Three out of nine Seyfert 1s (NGC 3516, 4151, and 4235) have spatially extended ionized gas regions within $1 \mathrm{kpc}$ or so around the nucleus. These regions have essentially the same morphology in both $\mathrm{H} \alpha+[\mathrm{N} \mathrm{II]}$ and [O III] emission. The remaining six (NGC 4051, 4639, $5033,5273,6814$, and 7450) show compact, star-like nuclear brightness profiles which are spatially unresolved to within the typical seeing disk $\left(1{ }^{\prime \prime} 5-2^{\prime \prime}\right)$. None of the Seyfert $1 \mathrm{~s}$ appear to have enhanced circumnuclear star formation within the inner kiloparsec or so from the nucleus.

Eight of the eleven Seyfert 2s (NGC 1068, 2110, 3081, 4388, 4941, 5283, 5347, and 5728) show extended ionized gas structures, some with quite complicated morphologies. Unlike the Seyfert $1 \mathrm{~s}$, the $\mathrm{H} \alpha+[\mathrm{N}$ II] and [O III] images show different (sometimes radically different) extended emission morphologies. In these, a basic pattern emerges: 
one-sided or two-sided distributions of high ionization emission-line gas. Maps of the [O III] $\lambda 5007 /(\mathrm{H} \alpha+[\mathrm{N} \mathrm{II}])$ emission ratio, which emphasize regions of high-ionization gas, reveal distinctly conical high-ionization regions with the nucleus at the apex in four of the Seyfert 2s (NGC 1068, 2110, 4388, and 5728). The remaining three (NGC 2273, 3982, and 4968) are compact, with no suggestion of marginally resolved nuclear sub-structure. Two (NGC 1068 and NGC 3982) show evidence of circumnuclear star formation regions that are sufficiently luminous to be considered "starbursts".

The extended regions in both $1 \mathrm{~s}$ and $2 \mathrm{~s}$ have spectra consistent with photoionization by a hard-photon spectrum, possibly the nuclear power-law continuum. Ionization by hot stars may be ruled out as no associated continuum structures are observed. For a few of the Seyfert $1 \mathrm{~s}$ and $2 \mathrm{~s}$ with extended emission regions, published radio maps are available. In every case, the radio continuum and optical emission-line structures share the same general alignment, but they are not strictly correlated knot-for-knot.

\section{Discussion}

The morphology and ionization of the extended regions suggests collimation of the nuclear ionizing radiation field, particularly in the Seyfert 2s. If a common feature of Seyfert 2s, it greatly complicates interpretation of their luminosity function. Ionizing radiation cones like those observed in four of the Seyfert $2 \mathrm{~s}$ are predicted by "unified" models of Seyfert nuclei in which Seyfert $2 \mathrm{~s}$ are Seyfert $1 \mathrm{~s}$ with the central source and broad line regions "hidden" from our line of sight, either by a thick accretion torus or dust clouds in the inner regions. For 3 of these there is secondary evidence favoring this interpretation: polarized broad H I lines in NGC 1068 (Antonucci and Miller 1985), weak broad H $\alpha$ lines in NGC 2110 (Shuder 1980), and NGC 4388 (Shields and Filippenko 1988). For the observed cone angles of $30-90^{\circ}$, the collimating material should intercept $70-95 \%$ of the total flux if the central source is radiating isotropically. If a dusty torus is responsible, larger FIR fluxes are predicted than are observed. A hot ion torus could work, the inner walls are an electron scattering atmosphere that could act as a crude mirror to redirect and collimate the central continuum.

However, there are problems with this simple "unified" model. Taken by themselves, the Seyfert $2 \mathrm{~s}$ are at first sight consistent with this picture. But, where extended emission is seen in Seyfert 1s, unlike that seen in 2s, it has the same $\mathrm{H} \alpha+[\mathrm{N} \mathrm{II}]$ and [O III] emission morphology. Thus, on the basis of the extended emission alone, one might guess the spectral type of the nucleus. None of the Seyfert $1 \mathrm{~s}$ observed look like pole-on versions of the extended emission Seyfert 2s. This is difficult to reconcile with the idea that these are the same basic objects viewed along different lines of sight. Either we are subject to subtle biases (e.g., a large range of central luminosities, accretion disk thickness, or amount circumnuclear gas), or it means that while some Seyfert 2 s are obscured Seyfert $1 \mathrm{~s}$, this is not true in general.

\section{References}

Antonucci, R.R.J., and Miller, J.S. 1985, Ap.J., 297, 621.

Pogge, R.W. 1988, Ph.D. Thesis, University of California.

Shields, J.C., and Filippenko, A.V. 1988, Ap.J.(Letters), 332, in press.

Shuder, J.M. 1980, Ap.J., 240, 32. 


\section{DISCUSSION}

ULRICH Several years ago Pequignot and I observed the extranuclear gas emission in NGC 3516 with the 4-m telescope at KPNO ( $A p . J ., 238,45,1980)$. We analyzed the line intensities in terms of photoionization by the non-thermal flux emitted by the nucleus. There is good agreement between our model and the observed line intensity ratios at 1 to several $\mathrm{kpc}$ from the nucleus. The gas density was obtained from the [S II] lines and the absolute ionizing flux was known from our IUE observations of NGC 3516.

POGGE That is correct. NGC 3516 is a Seyfert 1. For Seyfert 2s, the range of the apparent deficit of luminosity along the line of sight from the nucleus compared with what is needed from simple ionization arguments is a factor of a few (including 1.0) to about 100 . 